\title{
hsa_circ_0101119 facilitates the progression of cervical cancer via an interaction with EIF4A3 to inhibit TCEAL6 expression
}

\author{
XUEZUO SUI ${ }^{*}$, YANCHUN WANG ${ }^{*}$ and HUI LIU \\ Department of Gynaecology and Obstetrics, Yantai Hospital of \\ Traditional Chinese Medicine, Yantai, Shandong 264013, P.R. China
}

Received December 31, 2020; Accepted June 4, 2021

DOI: $10.3892 / \mathrm{mmr} .2021 .12293$

\begin{abstract}
Recently, circular RNAs (circRNAs/circs) have attracted increased attention due to their regulatory role in a variety of cancer types. However, the role and molecular mechanisms of circRNAs in cervical cancer (CC) remain unknown. The present study aimed to investigate the function of hsa_circ_0101119 on CC and its potential mechanisms. The differentially expressed circRNAs associated with CC were screened out using $\mathrm{R}$ software, according to the database of Gene Expression Omnibus (GEO). The expression levels of hsa_circ_0101119, eukaryotic initiation factor 4A-3 (EIF4A3) and transcription elongation factor A-like 6 (TCEAL6) in $\mathrm{CC}$ cells were detected via reverse transcription-quantitative (RT-q)PCR, and their expression levels in CC tissues were analyzed based on the database of GEO and the Cancer Genome Atlas. Moreover, the accurate functions of hsa_circ_0101119 and TCEAL6 on the proliferation, apoptosis, migration and invasion of SiHa and HeLa cells was examined using colony formation assay, 5-ethynyl-20-deoxyuridine incorporation assay, flow cytometry and Transwell assay. Next, the underlying mechanisms of hsa_circ_0101119 on CC progression were determined via bioinformatics analysis, RNA immunoprecipitation assay, RNA pull down assay, RT-qPCR and western blotting. It was found that hsa_circ_0101119 was highly expressed in CC tissues and cells, while TCEAL6 was lowly expressed. Knockdown of hsa_circ_0101119 or TCEAL6 overexpression significantly inhibited the proliferation, migration and invasion of SiHa and HeLa cells, but facilitated apoptosis. It was also demonstrated that hsa_circ_0101119 could recruit EIF4A3 to inhibit TCEAL6 expression in CC. Furthermore,
\end{abstract}

Correspondence to: Dr Hui Liu, Department of Gynaecology and Obstetrics, Yantai Hospital of Traditional Chinese Medicine, 39 Xingfu Road, Zhifu, Yantai, Shandong 264013, P.R. China

E-mail:1hlh2008@163.com

${ }^{*}$ Contributed equally

Key words: cervical cancer, hsa_circular RNA_0101119, eukaryotic initiation factor 4A-3, transcription elongation factor A-like 6, proliferation, metastasis knockdown of TCEAL6 could reverse the effects of silencing hsa_circ_0101119 on the proliferation, apoptosis, migration and invasion of HeLa cells. In conclusion, the present study revealed that hsa_circ_0101119 could facilitate cell proliferation, migration and invasion, and suppress apoptosis in CC via an interaction with EIF4A3 to inhibit TCEAL6 expression, which may provide a potential therapeutic target for $\mathrm{CC}$ treatment.

\section{Introduction}

Cervical cancer (CC) is one of the most common gynecological neoplasms among women worldwide (1). The incidence and mortality rates of CC have been increasing every year in developed and developing countries $(2,3)$. Despite the fact that encouraging improvements have been achieved in CC chemotherapy, radiotherapy and surgical therapies, the 5-year survival rate of patients with CC remains unsatisfactory (4). Therefore, it is essential to identify effective therapeutic targets and discover molecular mechanisms in CC progression.

Circular RNAs (circRNAs/circs), a novel class of non-coding RNAs, are featured with a continuous closed loop without 5'-3' polarity or a polyadenylated tail (5). In recent years, accumulating evidence has confirmed that circRNAs have significant implications in numerous tumors (6). circRNAs have been reported to be involved in the biological behaviors of tumor cells, including proliferation, survival, apoptosis and metastasis $(7,8)$. For example, hsa_circ_0000263 facilitates $\mathrm{CC}$ progression by regulating $\mathrm{CC}$ cell proliferation and migration (9). Ou et al (10) revealed that circ_angiomotin like 1 (AMOTL1) could promote CC growth by promoting AMOTL1 expression. Moreover, a study by Wang et al (11) reported that hsa_circ_0101119 expression was notably upregulated in the peripheral blood of patients with cervical squamous cell carcinoma. However, to the best of our knowledge, the role of hsa_circ_0101119 on CC has not yet been evaluated.

Recently, an increasing number of researchers have paid attention to the application of circRNAs in cancer diagnosis $(12,13)$. The main mechanisms via which circRNAs serve a role in cancer are by directly interacting with microRNAs (miRNAs) or RNA binding proteins (RBPs) $(8,14-16)$. Eukaryotic initiation factor 4A-3 (EIF4A3) is one of the RBPs that is a core component of the exon junction complex (17). The roles of EIF4A3 are reported to be complex and important 
in numerous diseases, and the abnormal structure and function of EIF4A3 directly lead to the changes of its downstream biological effects (18).

Members of the transcription elongation factor A-like (TCEAL) gene family contain TFA domains and may function as nuclear phosphoproteins that modulate transcription in a promoter context-dependent manner (19-21). At present, TCEAL7 is frequently deregulated in tumors, and its decreased expression often correlates with malignant clinical process and poor prognosis in multiple cancer types, including gastric cancer and non-small cell lung cancer $(22,23)$. Moreover, Chien et al (24) revealed that TCEAL6 has a sequence similarity to TCEAL7. Interestingly, TCEAL6 is reported to be lowly expressed in the early stage of CC (25). However, whether hsa_circ_0101119 affects the progression of CC via an interaction with EIF4A3 to regulate TCEAL6 expression remains unknown. The present study aimed to investigate the function of hsa_circ_0101119 in CC, and its regulatory mechanism that is associated with EIF4A3 and TCEAL6.

\section{Materials and methods}

Bioinformatics analysis. Gene expression data matrix (GSE102686) was derived from the Gene Expression Omnibus (GEO) database (https://www.ncbi.nlm.nih.gov/geo/), which included five $\mathrm{CC}$ samples and five adjacent non-tumorous samples (26). The present study identified the top five hsa_circRNAs with the highest differential expression, and selected hsa_circ_0101119 as the research target. Then, RNA-Protein Interaction Prediction (RPISeq; version 1.0; http://pridb.gdcb.iastate.edu/RPISeq/) was used to predict the interaction probabilities of RNA-binding protein EIF4A3 with hsa_circ_0101119 and TCEAL6.

Cell culture and transfection. The four human CC cell lines (C-33A, SiHa, CaSki and HeLa) and the normal human cervical epithelial cell line, HcerEpic, were supplied by VCANBIO Cell \& Gene Engineering Co., Ltd. All the cells were cultured in RPMI 1640 medium (Gibco; Thermo Fisher Scientific, Inc.) supplemented with $10 \%$ (v/v) FBS (Invitrogen; Thermo Fisher Scientific, Inc.) and $1 \%$ penicillin/streptomycin (Invitrogen; Thermo Fisher Scientific, Inc.), and cultured at $37^{\circ} \mathrm{C}$ in a humidified $5 \% \mathrm{CO}_{2}$ incubator.

Short hairpin (sh)RNA targeting EIF4A3 (sh-EIF4A3) and TCEAL6 (sh-TCEAL6), small interfering (si)RNA targeting hsa_circ_0101119 (si-hsa_circ), pcDNA3.1-TCEAL6 (pcDNA-TCEAL6) and their correspond negative controls (sh-NC, si-NC and pcDNA-NC) were purchased from Shanghai GenePharma Co., Ltd. The sh-NC was a plasmid containing a non-targeting (scramble) shRNA sequences, and the si-NC was a non-targeting (scramble) siRNA sequence. The sequences were as follows: Sh-EIF4A3, 5'-GGAAGACAT GACTAAAGTGGA-3'; sh-TCEAL6, 5'-GGAGAAGGGATC CGGTAGATT-3'; sh-NC, 5'-GGTAGTGGACGATGAGAC AGT-3'; si-hsa_circ, 5'-ATGAGCAGCCATACACTGCTT-3'; si-NC, 5'-GCTCTACTTCGACGACAAGAT-3'. According to the manufacturer's instruction, the cells were transfected with sh-EIF4A3 $(20 \mu \mathrm{l} / \mathrm{ml})$, sh-TCEAL6 $(20 \mu \mathrm{l} / \mathrm{ml})$, si-hsa_circ $(50 \mathrm{nM})$ or pcDNA-TCEAL6 $(4 \mu \mathrm{g})$ using Lipofectamine ${ }^{\circledR} 3000$ (Invitrogen; Thermo Fisher Scientific, Inc.) at $37^{\circ} \mathrm{C}$ for $48 \mathrm{~h}$.
Finally, the transfection efficiency was determined via reverse transcription-quantitative PCR (RT-qPCR).

Colony formation assay. The transfected SiHa and HeLa cells (500 cells/well) were seeded into 6 -well plates. Then, the cells were grown for 14 days at $37^{\circ} \mathrm{C}$. Next, $4 \%$ paraformaldehyde was used to fix colonies for $15 \mathrm{~min}$ at room temperature and $0.1 \%$ crystal violet was used to stain colonies for $30 \mathrm{~min}$ at room temperature. Finally, the numbers of colonies containing $>500$ cells were assessed using a light microscope (magnification, x100).

5-Ethynyl-20-deoxyuridine (EdU) incorporation assay. The EdU assay was carried out with the EdU labeling/detection kit (Guangzhou RiboBio Co., Ltd.) according to the manufacturer's instructions. The transfected $\mathrm{SiHa}$ and HeLa cells were incubated with $50 \mu \mathrm{M}$ EdU solution for $2 \mathrm{~h}$ at room temperature. Then, the cells were fixed with $4 \%$ paraformaldehyde for $30 \mathrm{~min}$ at room temperature, followed by permeabilized with $0.5 \%$ Triton X-100 at room temperature and stained with anti-EdU working solution for $30 \mathrm{~min}$ in the dark at room temperature. Finally, DAPI (Sigma-Aldrich; Merck KGaA) was used for staining the cell nucleus at room temperature for $30 \mathrm{~min}$, and the EdU-positive cells were observed using fluorescent microscopy (magnification, x200).

Flow cytometry. The apoptosis of SiHa and HeLa cells was detected using the Annexin V-PI kit (Beyotime Institute of Biotechnology). The transfected SiHa and HeLa cells were collected and resuspended in the binding buffer. Subsequently, the cells were labeled with Annexin V-FITC and PI. Finally, cell apoptosis was detected using a flow cytometry (FACSCalibur; BD Biosciences), and then data were analyzed using FlowJo software (version v7.6.5; FlowJo LLC).

Transwell assay. Transwell chambers with $8-\mu \mathrm{m}$ pores were obtained from Corning, Inc. A total of $48 \mathrm{~h}$ after transfection, the $\mathrm{SiHa}$ and HeLa cells were suspended into serum-free medium and seeded into the upper chamber without Matrigel (BD Biosciences). Next, $500 \mu 1$ medium with 10\% FBS was added to the lower chamber as the chemoattractant. After a 24-h incubation at room temperature, the migrated cells were fixed with methanol at $37^{\circ} \mathrm{C}$ for $30 \mathrm{~min}$ and stained with $0.1 \%$ crystal violet at $37^{\circ} \mathrm{C}$ for $30 \mathrm{~min}$, following which they were imaged and calculated using an inverted microscope (magnification, x100). Moreover, the cell invasion assay was performed at the same time with the aforementioned steps, except that the upper chamber was coated with Matrigel at room temperature overnight.

RNA immunoprecipitation (RIP) assay. The RIP assay was performed using an EZ-Magna RIP ${ }^{\mathrm{TM}}$ RNA kit (MilliporeSigma) to determine the endogenous interaction between EIF4A3 and hsa_circ_0101119 or TCEAL6, according to the manufacturer's protocols. Briefly, the cells were cross-linked by treating with formaldehyde for $10 \mathrm{~min}$ at room temperature. After washing with cold PBS, cells were incubated in $4 \mathrm{ml}$ cell lysis buffer for $15 \mathrm{~min}$ in ice. After nuclear extraction using a Dounce homogenizer (Wheaton; DWK Life Sciences), the chromatin was sheared by sonication 
(25\% power, $4.5 \mathrm{sec}$ impact, $9 \mathrm{sec}$ clearance, 14 times) at room temperature to obtain DNA fragments in a range between 1,000-200 bp. Following DNase treatment for $30 \mathrm{~min}$ at $37^{\circ} \mathrm{C}$, the cell lysates were incubated with RIP buffer containing the magnetic beads conjugated with anti-EIF4A3 antibody for $2 \mathrm{~h}$ at $4^{\circ} \mathrm{C}$ with gently shaking. The anti-IgG antibody served as the control. The immunoprecipitated RNA was released by the reverse cross-linking using proteinase $\mathrm{K}$ at $4^{\circ} \mathrm{C}$ overnight. Finally, the immunoprecipitated RNA was extracted using a RNeasy Min Elute Cleanup kit (Qiagen, Inc.) and then analyzed using an RT-qPCR assay.

RNA pull down assay. The interaction between hsa circ_0101119 and EIF4A3 was assessed using the Magnetic RNA-Protein Pull-Down kit (Pierce; Thermo Fisher Scientific, Inc.), following the manufacturer's instructions. Biotin-labeled hsa_circ_0101119 or antisense RNA, which was supplied by Genepharm, Inc., was co-incubated with the magnetic beads and the protein extract of $\mathrm{SiHa}$ or Hela cells. The pull-down protein was measured via western blotting.

$R T-q P C R$. Total RNA from cells was extracted using TRIzol ${ }^{\circledR}$ reagent (Invitrogen; Thermo Fisher Scientific, Inc.), according to the manufacturer's instructions. Subsequently, cDNA was synthesized and amplified from total RNA ( $2 \mu \mathrm{g})$ using the One Step PrimeScript cDNA Synthesis kit (Takara Bio, Inc.) according to the manufacturer's protocols. Then, cDNA samples $(1 \mu \mathrm{l})$ were subjected to a qPCR reaction system in presence of SYBR Premix Ex Taq II kit (Takara Bio, Inc.). GAPDH was used as an endogenous reference. All primers were designed by Shanghai GenePharma Co., Ltd. and were as follows: Hsa_circ_0101119 forward, 5'-AAGCACACCAGC TTCTCCTC-3' and reverse, 5'-GCGTGCTGGCATAGGATT TG-3'; EIF4A3 forward, 5'-CCCTCACCACAATGACAG CA-3' and reverse, 5'-TGACCCACGCAGGTTAAACA-3'; TCEAL6 forward, 5'-CAGCCGCCATTTCTTTCCAG-3' and reverse, 5'-GGAAACATCTGACCTCCGCA-3'; and GAPDH forward, 5'-GAAGGTGAAGGTCGGAGTC-3' and reverse, 5'-GAAGATGGTGATGGGATTTC-3'. The relative expression levels were calculated using the $2^{-\Delta \Delta \mathrm{Cq}}$ method (27). The RT-qPCR reaction conditions were as follows: Initial denaturation at $95^{\circ} \mathrm{C}$ for $3 \mathrm{~min}$, followed by 40 cycles of $95^{\circ} \mathrm{C}$ for $10 \mathrm{sec}, 60^{\circ} \mathrm{C}$ for $30 \mathrm{sec}$ and $72^{\circ} \mathrm{C}$ for $30 \mathrm{sec}$.

Western blot analysis. Total proteins from transfected $\mathrm{SiHa}$ and HeLa cells were extracted using the cell lysis buffer (Cell Signaling Technology, Inc.) with a protease inhibitor. An enhanced BCA protein assay kit (Beyotime Institute of Biotechnology) was used to quantify the protein concentration. The protein sample $(50 \mu \mathrm{g})$ was separated by $10 \%$ SDS-PAGE, electrophoretically transferred onto PVDF membranes (MilliporeSigma) and blocked with 5\% skimmed milk at room temperature for $2 \mathrm{~h}$. Then, the membrane was incubated with the primary antibody overnight at $4^{\circ} \mathrm{C}$ and then incubated with the HRP-labeled secondary antibody (anti-mouse IgG, cat. no. 7076, 1:5,000; anti-rabbit IgG, cat. no. 7074, 1:5,000) at room temperature for $2 \mathrm{~h}$. Finally, the bands were visualized using an ECL detection kit (Thermo Fisher Scientific, Inc.). The following antibodies were used: Bax (cat. no. 2772; 1:1,000), caspase-3 (cat. no. 14220; 1:1,000), Bcl-2 (cat. no. 3498; 1:1,000),
E-cadherin (cat. no. 3195; 1:500), N-cadherin (cat. no. 14215; 1:1,000), Vimentin (cat. no. 5741; 1:1,000) from Cell Signaling Technology, Inc.; MMP-2 (cat. no. ab92536; 1:1,000), MMP-9 (cat. no. ab228402; 1:500), EIF4A3 (cat. no. ab180573; 1:500) from Abcam; and TCEAL6 (cat. no. ag11732; 1:500) from ProteinTech Group, Inc. The density of the protein bands was semi-quantitated using ImageJ software (version V1.8.0; National Institutes of Health).

Statistical analysis. Data are presented as the mean \pm SEM of $\geq 3$ experiments. All data in this study were examined using SPSS 22.0 software (IBM, Corp.) and GraphPad Prism 8.0 software (GraphPad Software, Inc.). The differences between groups were assessed using an unpaired or paired Student's t-test or one-way ANOVA with a Tukey's post hoc test. The correlation between EIF4A3 and TCEAL6 was analyzed via Pearson correlation analysis. $\mathrm{P}<0.05$ was considered to indicate a statistically significant difference.

\section{Results}

hsa_circ_0101119 is highly expressed in CC tissues and cells. To investigate circRNA effects in CC, the most differentially expressed circRNAs were analyzed in GSE102686 (Fig. 1A). hsa_circ_0101119 was found to be the most significantly upregulated circRNA (Fig. 1A). Moreover, it was identified that the expression level of hsa_circ_0101119 was notably upregulated in CC cells and tissues (Fig. 1B and C), further suggesting that hsa_circ_0101119 was highly expressed in CC.

Knockdown of hsa_circ_0101119 inhibits the proliferation of SiHa and HeLa cells. As shown in Fig. 2A, RT-qPCR was used to verify the transfection efficiency of hsa_circ_0101119 knockdown. Then, the effects of hsa_circ_0101119 on CC cells were assessed using EdU and colony formation assays. The results of EdU assay demonstrated that hsa_circ_0101119 knockdown markedly reduced the proliferation of SiHa and HeLa cells (Fig. 2B). Moreover, colony formation assay results suggested that the cell clones of SiHa and HeLa cells were significantly decreased after knockdown of hsa_circ_0101119 (Fig. 2C). Collectively, the data indicated that the knockdown of hsa_circ_0101119 could inhibit the proliferation of SiHa and HeLa cells.

Knockdown of hsa_circ_0101119 facilitates the apoptosis of $\mathrm{SiHa}$ and HeLa cells. The results of flow cytometry demonstrated that the knockdown of hsa_circ_0101119 significantly increased the apoptosis of SiHa and HeLa cells (Fig. 3A). In addition, the expression levels of apoptosis-associated proteins (Bax, caspase-3 and Bcl-2) were detected via western blot analysis. As shown in Fig. 3B, the expression levels of Bax and caspase-3 in SiHa and HeLa cells were markedly upregulated after knockdown of hsa_circ_0101119, but Bcl-2 expression was significantly downregulated. These findings suggested that the knockdown of hsa_circ_0101119 could facilitate the apoptosis of SiHa and HeLa cells.

Knockdown of hsa_circ_0101119 inhibits the migration and invasion of SiHa and HeLa cells. Knockdown of hsa_circ_0101119 significantly decreased the migration and 

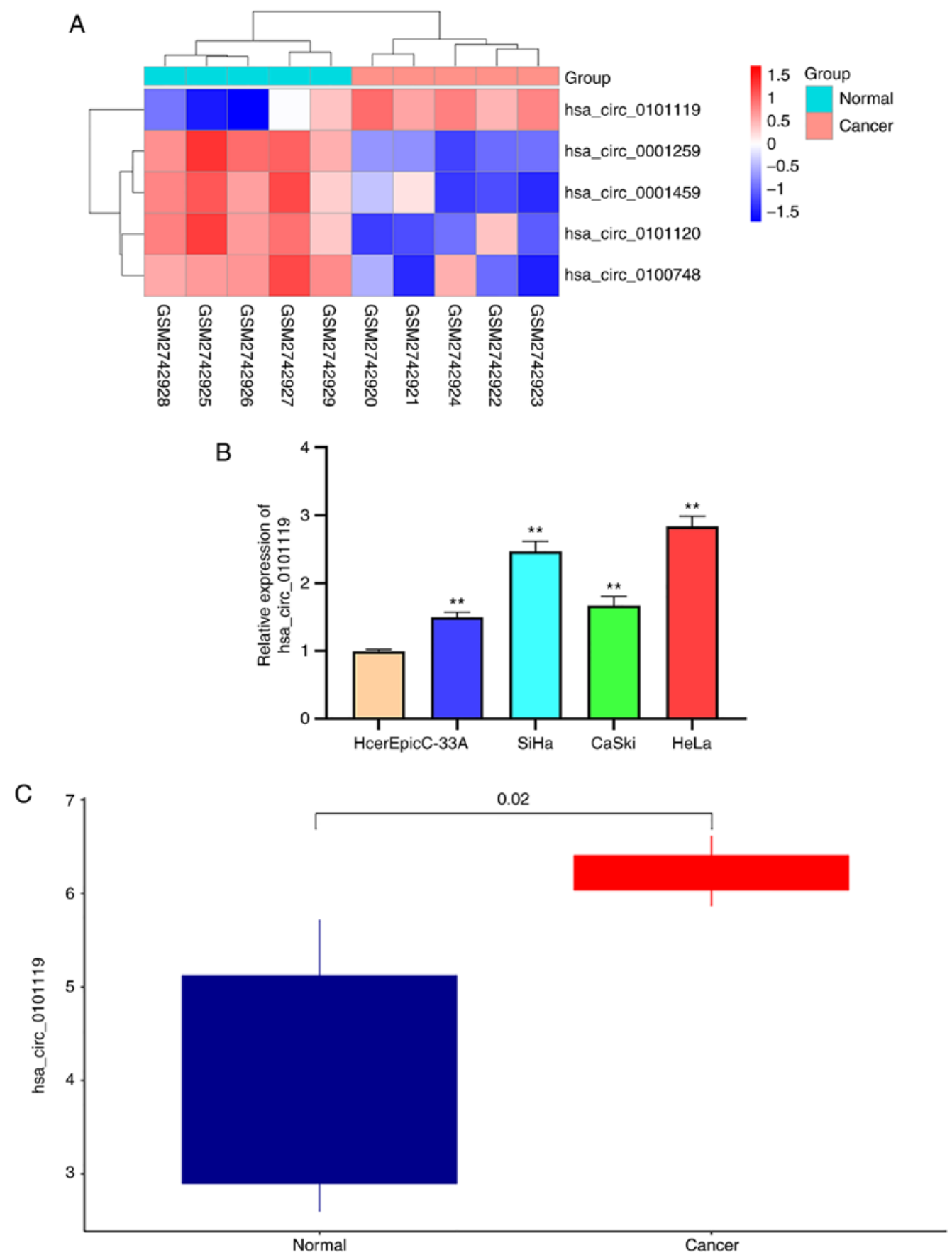

Figure 1. hsa_circ_0101119 is highly expressed in CC tissues and cells. (A) Heatmap of differentially expressed circRNAs in CC tissues and normal tissues according to the online data set (GSE102686). (B) Expression level of hsa_circ_0101119 was detected via reverse transcription-quantitative PCR in CC cell lines (C-33A, SiHa, CaSki and HeLa) and normal human cervical epithelial cell line, HcerEpic. ${ }^{* *} \mathrm{P}<0.01$ vs. HcerEpic cells. (C) Expression level of hsa_circ_0101119 in CC tissues and normal tissues, according to the online data set (GSE102686). circRNA/circ, circular RNA.

invasion of SiHa and HeLa cells (Fig. 4A and B). MMPs, such as MMP-2 and MMP-9, which are secreted by cells, are reported to promote cancer cell invasion and migration (28). Thus, the effects of hsa_circ_0101119 knockdown on migration and invasion were determined by detecting the expression levels of MMP-2 and MMP-9. The results demonstrated that the knockdown of hsa_circ_0101119 significantly reduced the expression levels of MMP-2 and MMP-9 in SiHa and HeLa cells (Fig. 4C).

The epithelial to mesenchymal transition has been reported to serve important roles in CC progression and metastasis (29).
The expression level of E-cadherin in SiHa and HeLa cells was markedly increased after knockdown of hsa_circ_0101119, but the expression levels of $\mathrm{N}$-cadherin and Vimentin were significantly decreased (Fig. 4C). These results indicated that the knockdown of hsa_circ_0101119 could inhibit the migration and invasion of SiHa and HeLa cells.

hsa_circ_0101119 recruits EIF4A3 to inhibit TCEAL6 expression in $C C$. The bioinformatics analysis revealed that hsa_circ_0101119 can bind to EIF4A3 (RF Classifier: 0.80; SVM Classifier: 0.77) (Fig. 5A). Using RIP and RNA pull 

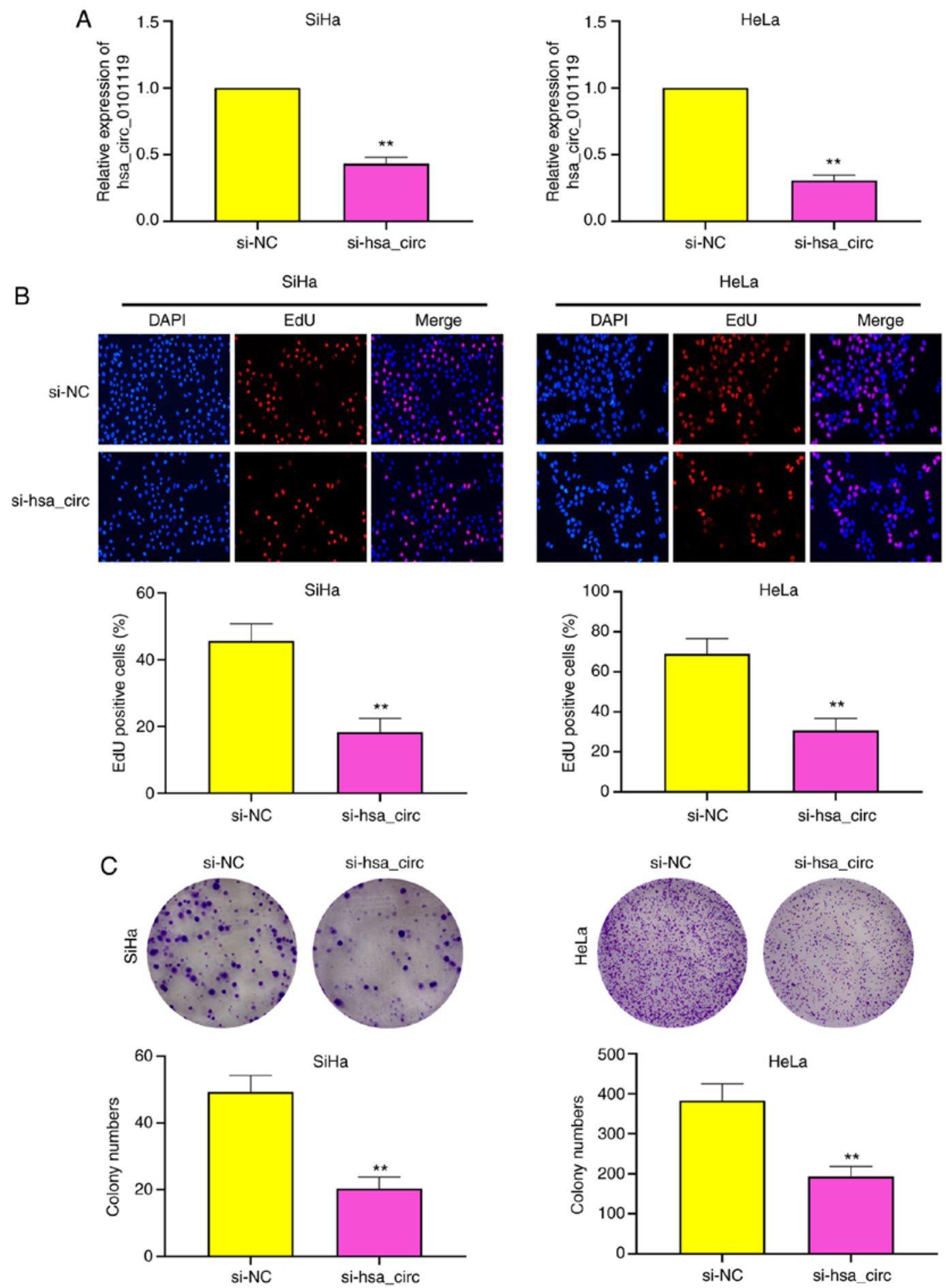

Figure 2. Knockdown of hsa_circ_0101119 inhibits the proliferation of SiHa and HeLa cells. (A) Expression level of hsa_circ_0101119 was detected via reverse transcription-quantitative PCR in transfected SiHa and HeLa cells. (B) Proliferation of SiHa and HeLa cells was determined using an EdU assay after transfection with si-hsa circ 0101119 (magnification, x200). (C) Colonies numbers of SiHa and HeLa cells were detected using a colony formation assay after transfection with si-hsa_circ_0101119 (magnification, x100). ${ }^{* *} \mathrm{P}<0.01$ vs. si-NC group. NC, negative control; si, small interfering RNA; circ, circular RNA; EdU, 5-ethynyl-20-deoxyuridine.

down assays, it was also identified that hsa_circ_0101119 could bind to EIF4A3 in SiHa and HeLa cells (Fig. 5B and C). To examine whether has_circ_0101119 could directly regulate TCEAL6 after binding with EIF4A3, bioinformatics analysis was conducted to predict the binding abundance between EIF4A3 and TCEAL6. The scores for RF Classifier and SVM Classifier were 0.80 and 0.60 , respectively (Fig. 5D), suggesting that EIF4A3 likely binds to TCEAL6. Moreover, the results of RIP assay verified this binding relationship (Fig. 5E).

It was found that EIF4A3 was highly expressed and TCEAL6 was lowly expressed in CC cells (Fig. 5F). Similarly, the analysis of The Cancer Genome Atlas (TCGA) dataset also indicated that EIF4A3 expression was significantly upregulated and TCEAL6 expression 
A
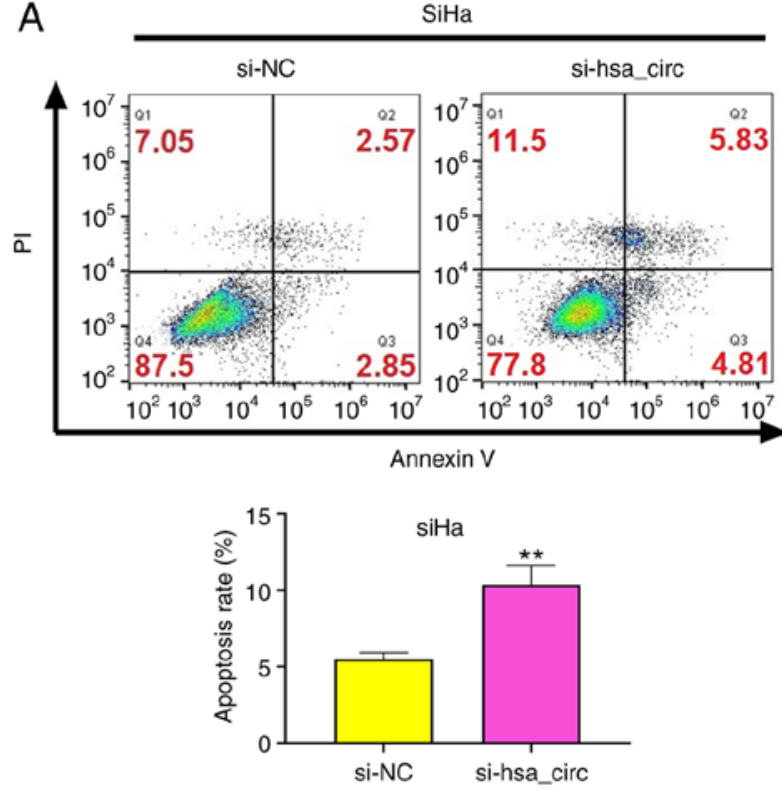
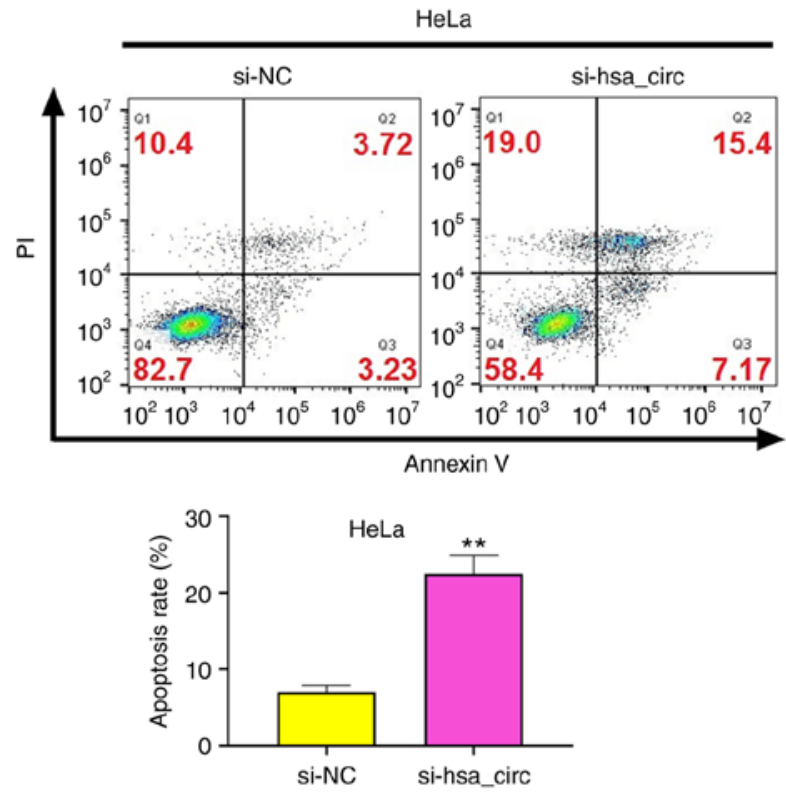

B

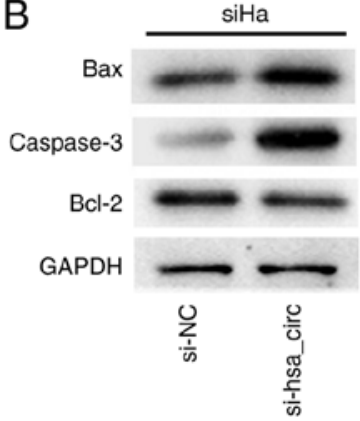

HeLa

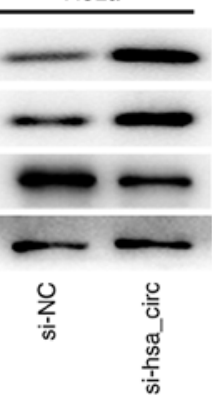

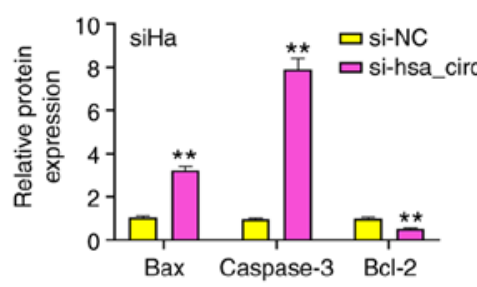

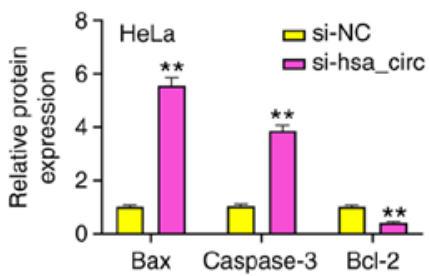

Figure 3. Knockdown of hsa_circ_0101119 facilitates the apoptosis of SiHa and HeLa cells. (A) After transfection with si-hsa_circ_0101119, flow cytometry was used to detect the apoptosis of SiHa and HeLa cells. (B) After transfection with si-hsa_circ_0101119, western blotting was performed to measure the expression levels of Bax, caspase-3 and Bcl-2 in SiHa and HeLa cells. ${ }^{* *} \mathrm{P}<0.01$ vs. si-NC group. NC, negative control; si, small interfering RNA; circ, circular RNA.

was downregulated in $\mathrm{CC}$ tissues (Fig. 5G). Moreover, the analysis of TCGA dataset revealed that EIF4A3 expression was negatively correlated with TCEAL6 expression in CC tissues (Fig. 5H).

As shown in Fig. 5I, RT-qPCR was used to verify the transfection efficiency of EIF4A3 knockdown. The results demonstrated that knockdown of EIF4A3 significantly increased the expression level of TCEAL6 in SiHa and HeLa cells (Fig. 5J), further suggesting that EIF4A3 expression was negatively associated with TCEAL6 expression in CC. In addition, it was found that knockdown of hsa_circ_0101119 significantly elevated TCEAL6 expression in SiHa and HeLa cells (Fig. 5K). Compared with the si-hsa_circ group, the co-transfection of si-hsa_circ and sh-EIF4A3 significantly increased the expression level of TCEAL6 in SiHa and HeLa cells (Fig. 5K). The schematic diagram depicts that hsa circ_0101119 regulates TCEAL6 expression by enhancing the binding of EIF4A3 to TCEAL6 mRNA, which inhibits TCEAL6 translation (Fig. 5L).

TCEAL6 overexpression inhibits the proliferation, migration and invasion, and promotes the apoptosis of SiHa and HeLa cells. As shown in Fig. 6A, RT-qPCR was used to verify the transfection efficiency of TCEAL6 overexpression. To verify the effect of TCEAL6 overexpression, colony formation assays (Fig. 6B), flow cytometry (Fig. 6C) and Transwell assays (Fig. 6D and E) were performed. The results indicated that TCEAL6 overexpression significantly inhibited the proliferation, migration and invasion, while it promoted the apoptosis of $\mathrm{SiHa}$ and HeLa cells (Fig. 6B-E).

Knockdown of TCEAL6 reverses the effects of hsa circ_0101119 knockdown on the proliferation, apoptosis, migration and invasion of HeLa cells. As presented in Fig. 7A, the transfection efficiency of sh-TCEAL6 was assessed via RT-qPCR. The results demonstrated that the proliferation, cell clones, migration and invasion of HeLa cells were significantly reduced in the si-hsa_circ + sh-NC group compared with the si-NC + sh-NC or si-hsa_circ + sh-TCEAL6 groups, while apoptosis was significantly elevated (Fig. 7B-E). In addition, compared with the si-NC + sh-NC or si-hsa_circ + sh-TCEAL6 groups, the proliferation, cell clones, migration and invasion of HeLa cells were significantly increased in the si-NC + sh-TCEAL6 group, but apoptosis was significantly decreased (Fig. 7B-E). These results indicated that the knockdown of TCEAL6 could reverse the effects of hsa_circ_0101119 knockdown on the proliferation, apoptosis, migration and invasion in HeLa cells. 
A
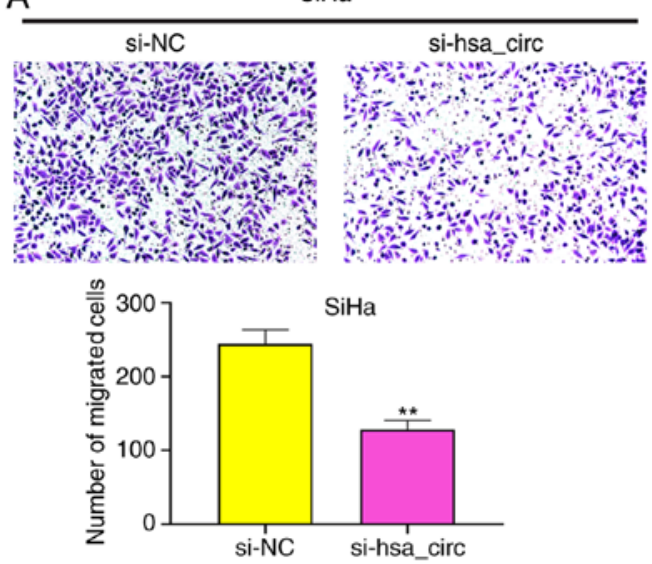

B
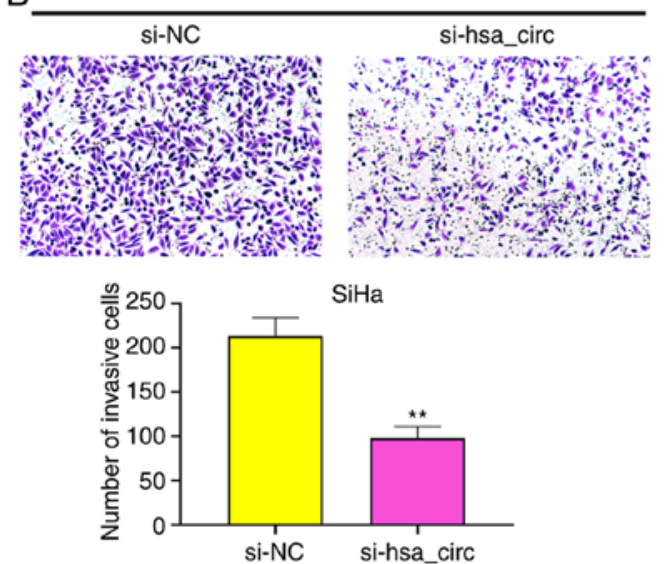

C

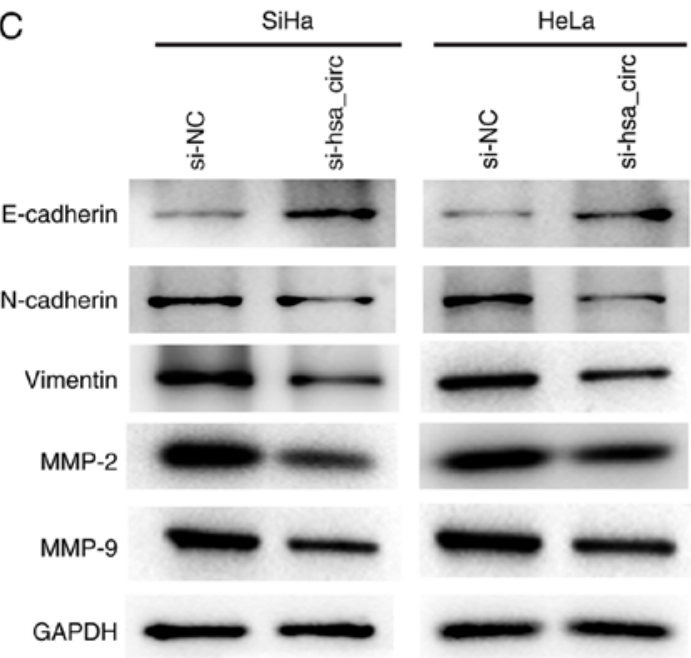

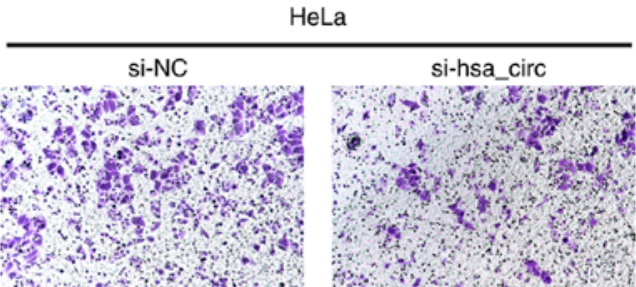
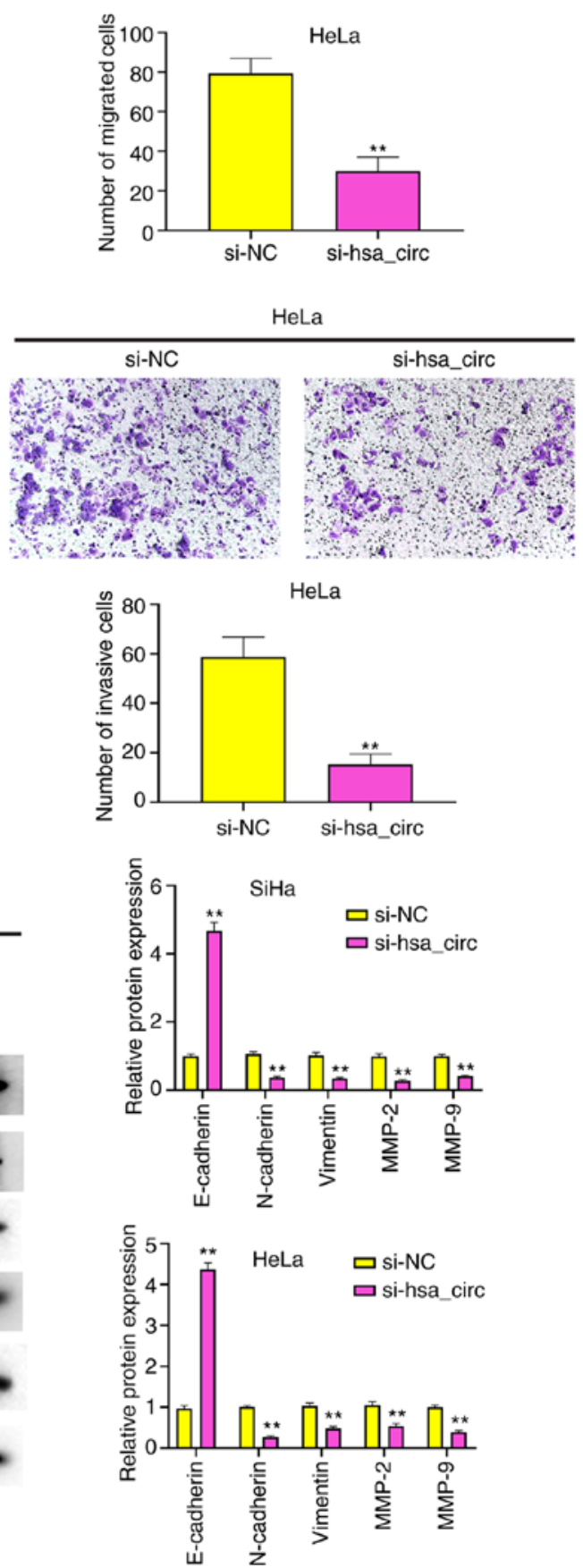

Figure 4. Knockdown of hsa_circ_0101119 inhibits the migration and invasion of SiHa and HeLa cells. (A) The migration of SiHa and HeLa cells was determined using a Transwell assay after transfection with si-hsa circ 0101119 (magnification, x100). (B) The invasion of SiHa and HeLa cells was determined using Transwell assay after transfection with si-hsa_circ_0101119 (magnification, x100). (C) After transfection with si-hsa_circ_0101119, western blotting was performed to measure the expression levels of E-cadherin, N-cadherin, Vimentin, MMP-2 and MMP-9 in SiHa and HeLa cells. "* P $<0.01$ vs. si-NC group. NC, negative control; si, small interfering RNA; circ, circular RNA.

\section{Discussion}

According to public reports, $>450,000$ patients are diagnosed with CC and 266,000 individuals die of CC every year $(30,31)$.
Although different strategies have been used for the treatment of CC, CC remains a health problem. Therefore, it is essential to identify effective therapeutic targets and discover the underlying molecular mechanisms in CC progression. In the 


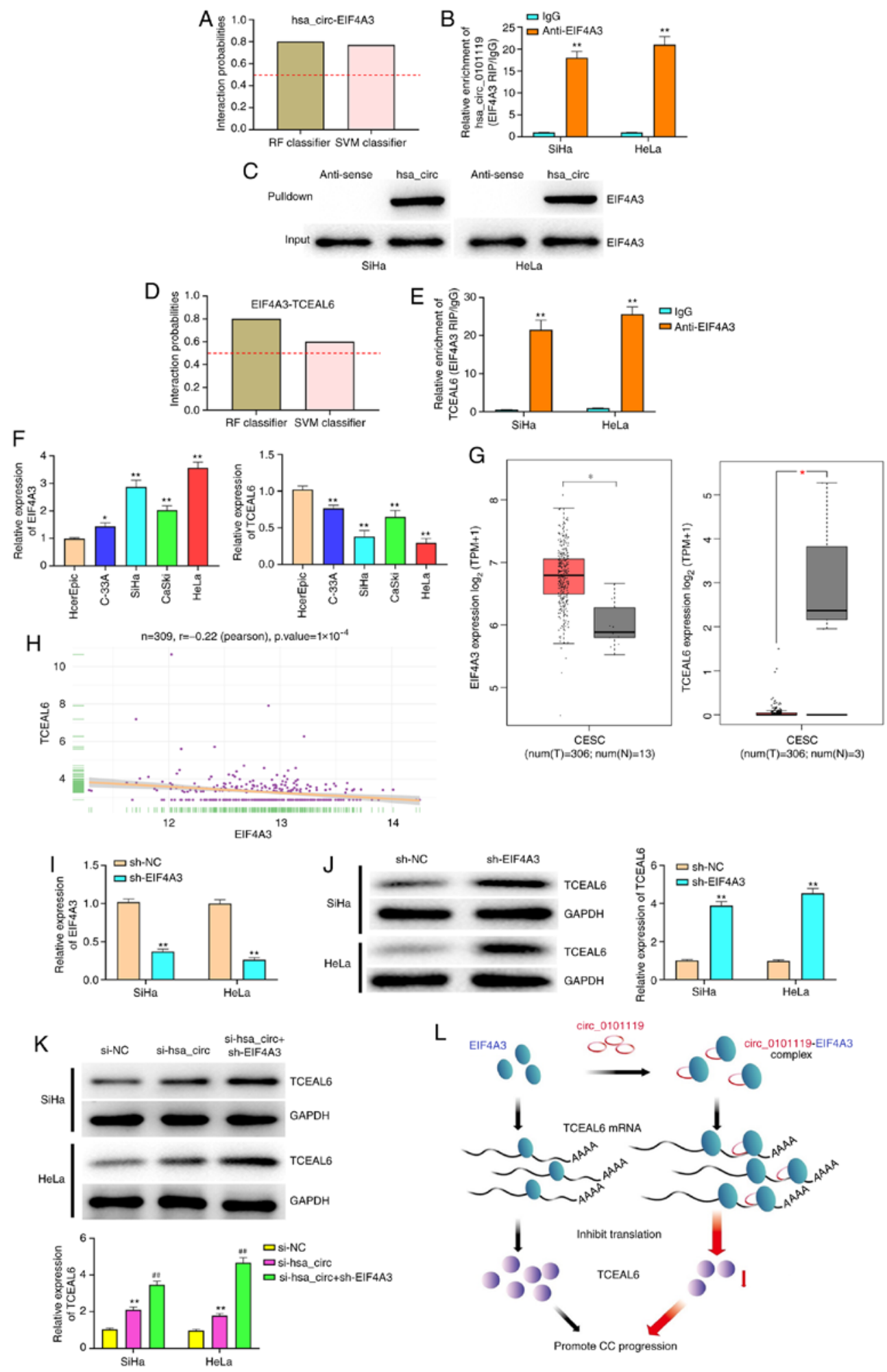

Figure 5. hsa_circ_0101119 recruits EIF4A3 to inhibit TCEAL6 expression in CC. (A) Bioinformatics was used to predict the interaction probabilities of the RNA-binding protein EIF4A3 with hsa_circ_0101119. Predictions with probabilities $>0.5$ were considered 'positive', suggesting that the corresponding RNA and protein are likely to interact. (B) RIP assay using anti-EIF4A3 showed that EIF4A3 precipitated hsa_circ_0101119 in SiHa and HeLa cell lysates. (C) Pull down assay indicated that biotin-labeled hsa_circ_0101119 interacted with EIF4A3. (D) Bioinformatics was used to predict the interaction probabilities of EIF4A3 with TCEAL6. (E) RIP assay using anti-EIF4A3 showed that EIF4A3 precipitated TCEAL6 in SiHa and HeLa cell lysates. (F) Expression levels of EIF4A3 and TCEAL6 were detected via RT-qPCR in CC cell lines (C-33A, SiHa, CaSki and HeLa) and a normal human cervical epithelial cell line, HcerEpic. (G) Expression levels of EIF4A3 and TCEAL6 in CC tissues and normal tissues, according to the analysis of TCGA. (H) Correlation between EIF4A3 and TCEAL6 in CC samples from TCGA. (I) After transfection with sh-EIF4A3, RT-qPCR was used to detect EIF4A3 expression in SiHa and HeLa cells. (J) After transfection with sh-EIF4A3, western blotting was performed to detect the expression level of TCEAL6 in SiHa and HeLa cells. (K) After co-transfection with si-hsa_circ_0101119 and sh-EIF4A3, western blotting was performed to measure the expression level of TCEAL6 in SiHa and HeLa cells. (L) A proposed model whereby hsa_circ_0101119 sequesters EIF4A3 away from TCEAL6 mRNA, in turn suppressing TCEAL6 mRNA translation. ${ }^{* *} \mathrm{P}<0.01$ vs. IgG group $(\mathrm{B}$ and $\mathrm{E})$; ${ }^{*} \mathrm{P}<0.05$, ${ }^{* * *} \mathrm{P}<0.01$ vs. HcerEpic cells group $(\mathrm{F})$; ${ }^{*} \mathrm{P}<0.05$ vs. normal tissues group (G); *" $\mathrm{P}<0.01$, vs. sh-NC group (I and J); ${ }^{* *} \mathrm{P}<0.01$ vs. sh-NC group, ${ }^{\# \#} \mathrm{P}<0.01$, vs. si-hsa_circ group. (K) RIP, RNA immunoprecipitation; RT-qPCR, reverse transcription-quantitative PCR; TCGA, The Cancer Genome Atlas; sh, short hairpin RNA; NC, negative control; si, small interfering RNA; circ, circular RNA; EIF4A3, eukaryotic initiation factor 4A-3; TCEAL6, transcription elongation factor A-like 6; T, tumor; N, normal; CC, cervical cancer. 

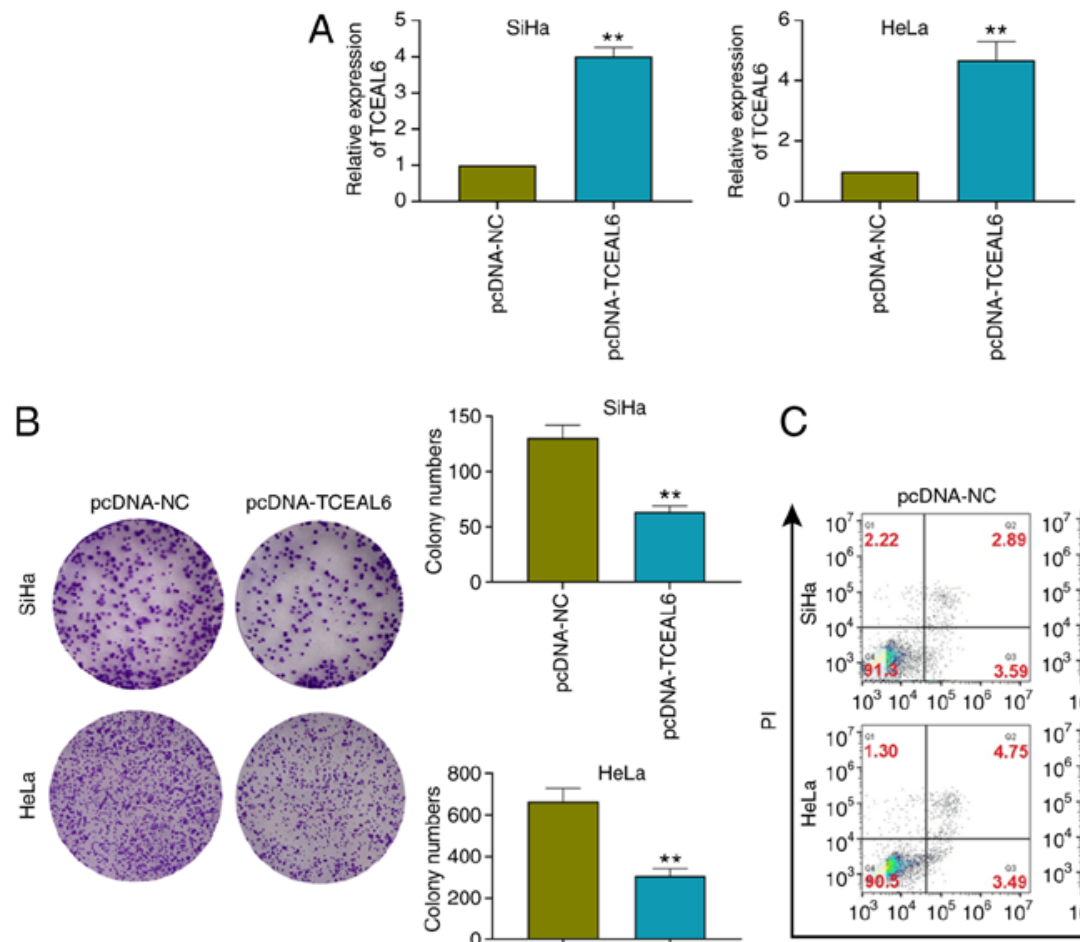

C
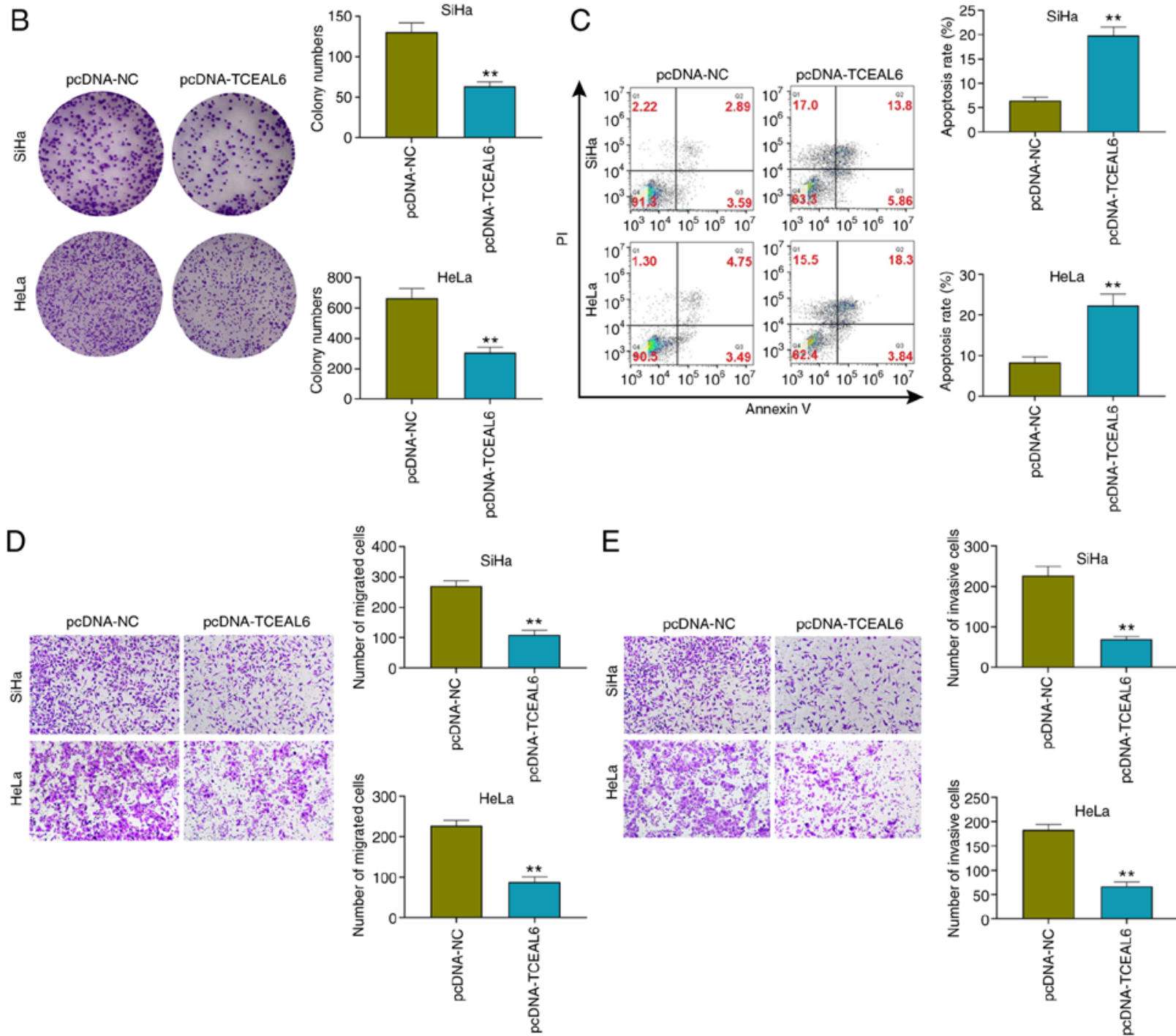

$\mathrm{E}$
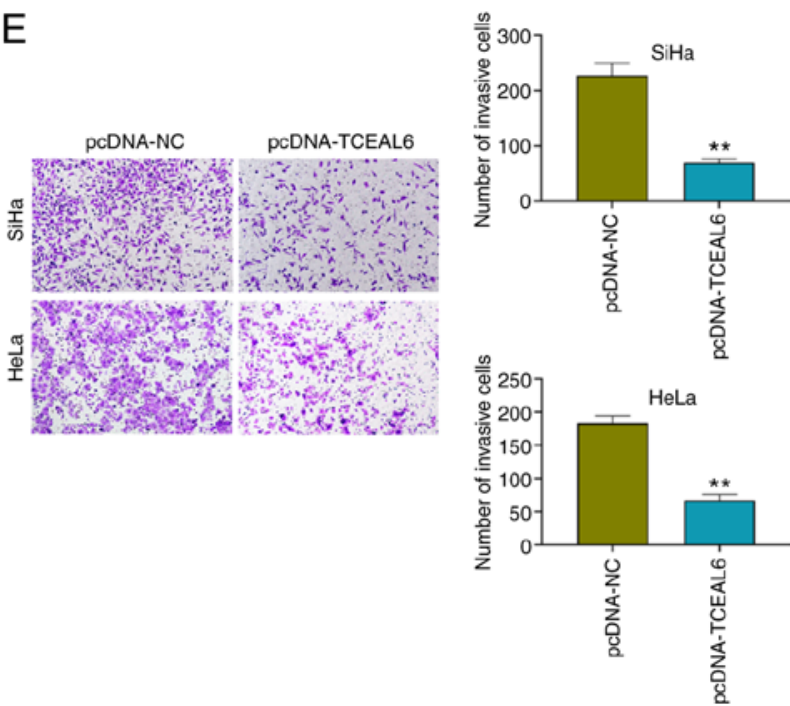

Figure 6. TCEAL6 overexpression inhibits proliferation, migration and invasion, and promotes apoptosis in SiHa and HeLa cells. (A) Expression level of TCEAL6 was detected via reverse transcription-quantitative PCR in transfected SiHa and HeLa cells. (B) After transfection with pcDNA-TCEAL6, colony numbers of SiHa and HeLa cells were detected using a colony formation assay (magnification, x100). (C) After transfection with pcDNA-TCEAL6, flow cytometry was used to detect the apoptosis of SiHa and HeLa cells. After transfection with pcDNA-TCEAL6, the (D) migration and (E) invasion of SiHa and HeLa cells was determined using a Transwell assay (magnification, $\mathrm{x} 100$ ). ${ }^{* *} \mathrm{P}<0.01$ vs. pcDNA-NC group. NC, negative control; TCEAL6, transcription elongation factor A-like 6.

present study, it was demonstrated that hsa_circ_0101119 could facilitate cell proliferation, migration and invasion, and suppress apoptosis in CC via an interaction with EIF4A3 to inhibit TCEAL6 expression.

Accumulating evidence has indicated that the dysregulation of circRNAs is associated with the occurrence and development of malignancies $(32,33)$. For example, the expression level of circ_102231 is upregulated in lung adenocarcinoma tissues (34). Zhang et al (35) reported that hsa_circ_0023404 was highly expressed and exerted an oncogenic role in CC. By analyzing the GSE102686 chip, the present study identified that the expression level of hsa_circ_0101119 was significantly upregulated in CC tissues compared with that in normal tissues. In addition, it was demonstrated that hsa_circ_0101119 expression was markedly increased in CC cells, which was in line with a previous study by Wang et al (11). It has also been revealed 
A
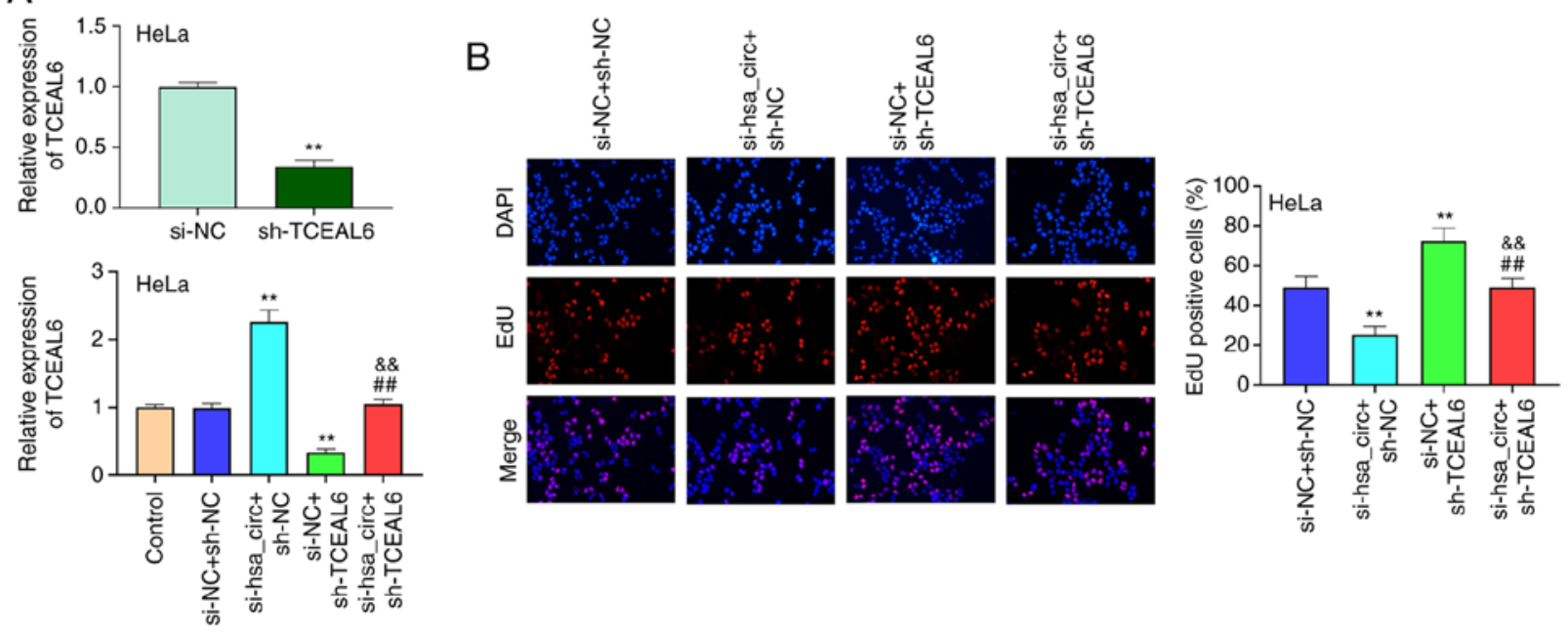

C
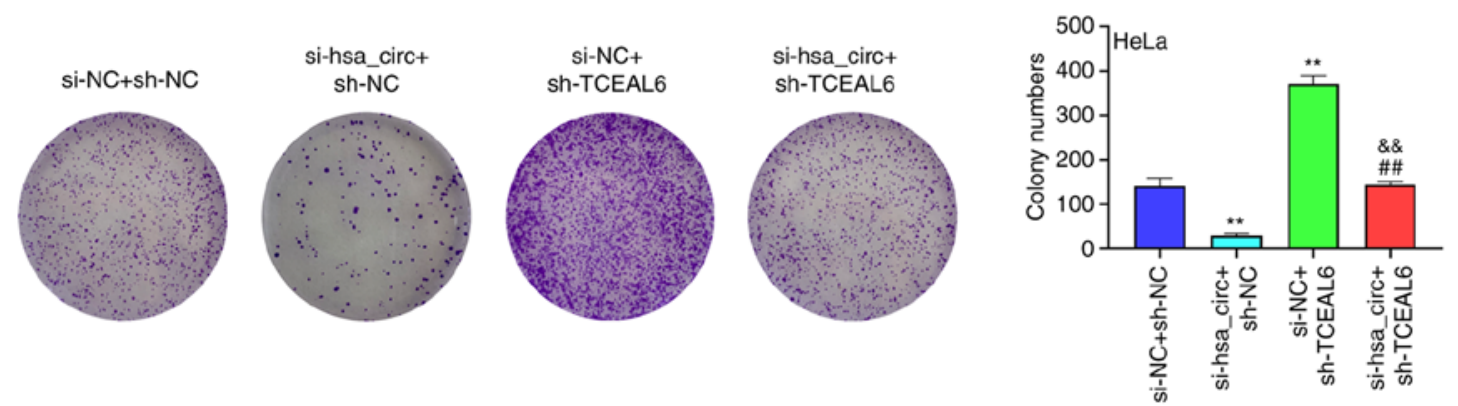

D
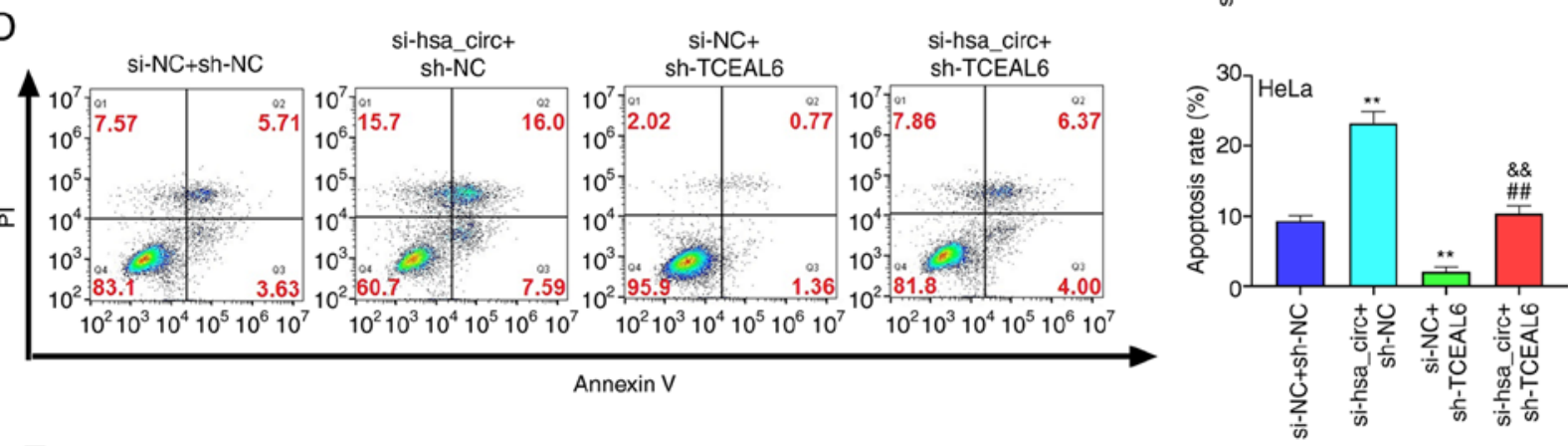

E
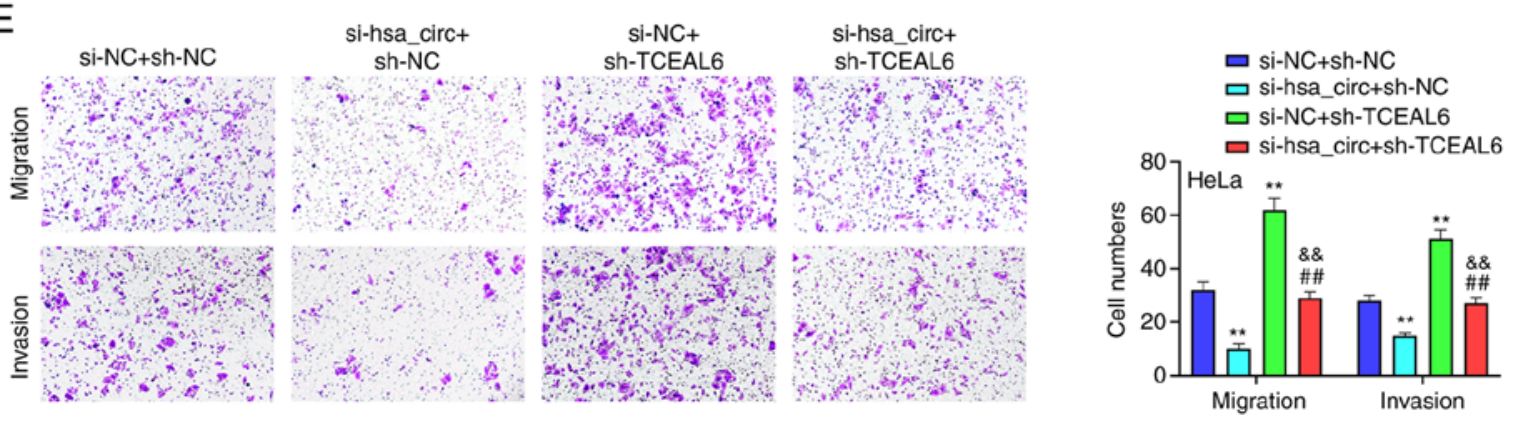

Figure 7. Knockdown of TCEAL6 reverses the effects of silencing hsa_circ_0101119 on the proliferation, apoptosis and migration and invasion in HeLa cells. (A) After transfection, the expression level of TCEAL6 was detected via reverse transcription-quantitative PCR in HeLa cells. (B) After co-transfection with si-hsa_circ_0101119 and sh-TCEAL6, HeLa cell proliferation was determined using an EdU assay (magnification, x200). (C) After co-transfection with si-hsa_circ_0101119 and sh-TCEAL6, the colony numbers of HeLa cells were detected with a colony formation assay (magnification, x100). (D) After co-transfection with si-hsa_circ_0101119 and sh-TCEAL6, flow cytometry was used to detect HeLa cell apoptosis. (E) After co-transfection with si-hsa_circ_0101119 and sh-TCEAL6, the migration and invasion of HeLa cells were determined using a Transwell assay (magnification, x 100). ${ }^{* *} \mathrm{P}<0.01 \mathrm{vs}$. si-NC + sh-NC group; ${ }^{\# \#} \mathrm{P}<0.01$ vs. si-hsa_circ + sh-NC; \&\& $\mathrm{P}<0.01$ vs. si-NC + sh-TCEAL6 group. sh, short hairpin RNA; NC, negative control; si, small interfering RNA; circ, circular RNA; TCEAL6, transcription elongation factor A-like 6; EdU, 5-ethynyl-20-deoxyuridine; circ, circular RNA.

that circRNAs can serve a critical role in different biological processes, including proliferation, survival, apoptosis and metastasis (36). For instance, circ-MYB proto-oncogene like 2 is reported to facilitate the proliferation and invasion of $\mathrm{CC}$ cells (37). Moreover, Chen et al (38) suggested that circRNA myosin light chain kinase could accelerate cell proliferation 
and repress apoptosis via the upregulation of Ras homolog, mTORC1 binding and the activation of the mTOR pathway in CC. Wang et al (39) also revealed that the overexpression of hsa_circ_0001038 could promote cell proliferation and invasion by regulating cyclin-M3 and metastasis-associated in colon cancer 1 expression in CC. In the present study, it was demonstrated that the knockdown of hsa_circ_0101119 could significantly inhibit the proliferation, migration and invasion, as well as facilitate the apoptosis of SiHa and HeLa cells.

Besides miRNAs, circRNAs can bind RBPs, modulate their availability in the cells and affect the post-transcriptional fates of RBP-interacting mRNAs $(5,40)$. The RBP EIF4A3 is reported to be an important regulator of post-transcriptional regulation processes, including mRNA splicing, transport, translation and surveillance (18). EIF4A3 has been shown to be upregulated and identified as a diagnostic marker in some cancer types, including breast cancer and lung cancer (18). Moreover, repression of EIF4A3 could affect the expression levels of transcripts associated with the cell cycle in cancer cells (41). In the recent years, numerous studies have shown that circRNAs serve vital roles in the development of cancer via binding EIF4A3. For example, circ_septin9 could significantly promote proliferation, migration and invasion, as well as inhibit apoptosis and autophagy in triple-negative breast cancer cells via binding EIF4A3 (42). Xu et al (43) also reported that circ_chromosome segregation 1 can inhibit colorectal cancer cell proliferation by binding to EIF4A3. Furthermore, circ_nectin cell adhesion molecule 3 was found to facilitate the proliferation of gastric cancer cells by combining with EIF4A3 (44). Using bioinformatics analysis, the current study predicted that hsa_circ_0101119 could bind to EIF4A3, and this binding of hsa_circ_0101119 to EIF4A3 was confirmed using RIP and RNA pull down assays.

Accumulating evidence has suggested that RBPs can conduct their roles by interacting with different types of target RNAs and forming ribonucleoprotein complexes $(45,46)$. The current bioinformatics analysis also identified a high binding abundance between EIF4A3 and TCEAL6. In the present study, it was demonstrated that EIF4A3 significantly negatively regulated TCEAL6 in CC cells. Biewenga et al (25) reported that TCEAL6 was lowly expressed in the early stage of $\mathrm{CC}$ and could serve as a potential biomarker for $\mathrm{CC}$, which was confirmed by the current experiments. Moreover, the present study identified that hsa_circ_0101119 could recruit EIF4A3 to inhibit TCEAL6 expression in CC, as determined via bioinformatics analysis, RIP assay, pull down assay, RT-qPCR and western blotting. To confirm this conclusion, the effects of co-transfection of si-hsa_circ_0101119 and sh-TCEAL6 on proliferation, apoptosis, migration and invasion were detected in HeLa cells. The data revealed that the knockdown of TCEAL6 could reverse the effects of silencing hsa_circ_0101119 on the proliferation, apoptosis, migration and invasion of HeLa cells. Of course, further studies are required to identify the effect of hsa_circ_0101119 and its underlying molecular mechanisms in $\mathrm{CC}$ in vivo.

In conclusion, the present study identified an oncogenic role of hsa_circ_0101119 in CC progression. It was demonstrated that hsa_circ_0101119 could facilitate cell proliferation, migration and invasion, and suppress apoptosis in $\mathrm{CC}$ via an interaction with EIF4A3 to inhibit TCEAL6 expression. These findings suggested that hsa_circ_0101119 may be an effective therapeutic target for $\mathrm{CC}$ treatment.

\section{Acknowledgements}

Not applicable.

\section{Funding}

No funding was received.

\section{Availability of data and materials}

The datasets used and/or analyzed during the current study are available from the corresponding author on reasonable request.

\section{Authors' contributions}

XS designed the study and performed the research. YW and HL analyzed data, and wrote the paper. All authors have read and approved the final manuscript, and met the authorship requirements stated earlier in this document, and each author believes that the manuscript represents honest work. All authors confirmed the authenticity of the raw data.

\section{Ethics approval and consent to participate}

Not applicable.

\section{Patient consent for publication}

Not applicable.

\section{Competing interests}

The authors declare that they have no competing interests.

\section{References}

1. Jemal A, Bray F, Center MM, Ferlay J and Forman D: Global cancer statistics. CA Cancer J Clin 61: 69-90, 2011.

2. Siegel RL, Miller KD and Jemal A: Cancer statistics, 2019. CA Cancer J Clin 69: 7-34, 2019.

3. Kessler TA: Cervical cancer: Prevention and early detection. Semin Oncol Nurs 33: 172-183, 2017.

4. Wright JD, Chen L, Tergas AI, Burke WM, Hou JY, Neugut AI, Ananth CV and Hershman DL: Population-level trends in relative survival for cervical cancer. Am J Obstet Gynecol 213: 670. e1-e7, 2015.

5. Hentze MW and Preiss T: Circular RNAs: Splicing's enigma variations. EMBO J 32: 923-925, 2013.

6. Han B, Chao J and Yao H: Circular RNA and its mechanisms in disease: From the bench to the clinic. Pharmacol Ther 187: 31-44, 2018.

7. Salzman J: Circular RNA expression: Its potential regulation and function. Trends Genet 32: 309-316, 2016.

8. Ebbesen KK, Kjems J and Hansen TB: Circular RNAs: Identification, biogenesis and function. Biochim Biophys Acta 1859: 163-168, 2016

9. Cai H, Zhang P, Xu M, Yan L, Liu N and Wu X: Circular RNA hsa_circ_0000263 participates in cervical cancer development by regulating target gene of miR-150-5p. J Cell Physiol 234: 11391-11400, 2019.

10. Ou R, Lv J, Zhang Q, Lin F, Zhu L, Huang F, Li X, Li T, Zhao L, Ren Y and Xu Y: circAMOTL1 Motivates AMOTL1 expression to facilitate cervical cancer growth. Mol Ther Nucleic Acids 19: 50-60, 2020. 
11. Wang YM, Huang LM, Li DR, Shao JH, Xiong SL, Wang CM and Lu SM: Hsa_circ_0101996 combined with hsa_circ_0101119 in peripheral whole blood can serve as the potential biomarkers for human cervical squamous cell carcinoma. Int J Clin Exp Pathol 10: 11924-11931, 2017.

12. Mao Y, Zhang L and Li Y: circEIF4G2 modulates the malignant features of cervical cancer via the miR-218/HOXA1 pathway. Mol Med Rep 19: 3714-3722, 2019.

13. Song T, Xu A, Zhang Z, Gao F, Zhao L, Chen X, Gao J and Kong X: CircRNA hsa_circRNA_101996 increases cervical cancer proliferation and invasion through activating TPX2 expression by restraining miR-8075. J Cell Physiol 234: 14296-14305, 2019.

14. Jeck WR and Sharpless NE: Detecting and characterizing circular RNAs. Nat Biotechnol 32: 453-461, 2014.

15. He JH, Li YG, Han ZP, Zhou JB, Chen WM, Lv YB, He ML Zuo JD and Zheng L: The CircRNA-ACAP2/Hsa-miR-21-5p/ tiam1 regulatory feedback circuit affects the proliferation migration, and invasion of colon cancer SW480 cells. Cell Physiol Biochem 49: 1539-1550, 2018

16. Chaichian S, Shafabakhsh R, Mirhashemi SM, Moazzami B and Asemi Z: Circular RNAs: A novel biomarker for cervical cancer. J Cell Physiol 235: 718-724, 2020.

17. Hauer C, Curk T, Anders S, Schwarzl T, Alleaume AM, Sieber J, Hollerer I, Bhuvanagiri M, Huber W, Hentze MW and Kulozik AE: Improved binding site assignment by high-resolution mapping of RNA-protein interactions using iCLIP. Nat Commun 6: 7921, 2015

18. Lin Y, Zhang J, Cai J, Liang R, Chen G, Qin G, Han X, Yuan C, Liu Z, Li Y, et al: Systematic analysis of gene expression alteration and co-expression network of eukaryotic initiation factor $4 \mathrm{~A}-3$ in CANcer. J Cancer 9: 4568-4577, 2018.

19. Yeh CH and Shatkin AJ: A HeLa-cell-encoded p21 is homologous to transcription elongation factor SII. Gene 143: 285-287, 1994.

20. Yeh $\mathrm{CH}$ and Shatkin AJ: Down-regulation of rous sarcoma virus long terminal repeat promoter activity by a hela cell basic protein. Proc Natl Acad Sci USA 91: 11002-11006, 1994.

21. Pillutla RC, Shimamoto A, Furuichi Y and Shatkin AJ: Genomic structure and chromosomal localization of TCEAL1, a human gene encoding the nuclear phosphoprotein p21/SIIR. Genomics 56: 217-220, 1999.

22. Huang CY, Chen YM, Zhao JJ, Chen YB, Jiang SS, Yan SM, Zhao BW, Pan K, Wang DD, Lv L, et al: Decreased expression of transcription elongation factor A-like 7 is associated with gastric adenocarcinoma prognosis. PLoS One 8: e54671, 2013.

23. Orhan C, Bulut P, Dalay N, Ersen E and Buyru N: Downregulation of TCEAL7 expression induces CCND1 expression in non-small cell lung cancer. Mol Biol Rep 46: 5251-5256, 2019.

24. Chien J, Staub J, Avula R, Zhang H, Liu W, Hartmann LC, Kaufmann SH, Smith DI and Shridhar V: Epigenetic silencing of TCEAL7 (Bex4) in ovarian cancer. Oncogene 24: 5089-5100, 2005.

25. Biewenga P, Buist MR, Moerland PD, Ver Loren van Themaat E, van Kampen AH, ten Kate FJ and Baas F: Gene expression in early stage cervical cancer. Gynecol Oncol 108: 520-526, 2008.

26. Jiao J, Zhang T, Jiao X, Huang T, Zhao L, Ma D and Cui B hsa_circ_0000745 promotes cervical cancer by increasing cell proliferation, migration, and invasion. J Cell Physiol 235: 1287-1295, 2020.

27. Livak KJ and Schmittgen TD: Analysis of relative gene expression data using real-time quantitative PCR and the 2(-Delta Delta C(T)) method. Methods 25: 402-408, 2001

28. Roomi MW, Monterrey JC, Kalinovsky T, Rath $M$ and Niedzwiecki A: Inhibition of invasion and MMPs by a nutrient mixture in human cancer cell lines: A correlation study. Ex Oncol 32: 243-248, 2010

29. Qureshi R, Arora H and Rizvi MA: EMT in cervical cancer: Its role in tumour progression and response to therapy. Cancer Lett 356: 321-331, 2015.
30. Torre LA, Bray F, Siegel RL, Ferlay J, Lortet-Tieulent J and Jemal A: Global cancer statistics, 2012. CA Cancer J Clin 65: 87-108, 2015.

31. Denny L: Cervical cancer: Prevention and treatment. Discov Med 14: 125-131, 2012.

32. Dragomir M and Calin GA: Circular RNAs in cancer-lessons learned from microRNAs. Front Oncol 8: 179, 2018.

33. Li M, Ding W, Sun T, Tariq MA, Xu T, Li P and Wang J: Biogenesis of circular RNAs and their roles in cardiovascular development and pathology. FEBS J 285: 220-232, 2018.

34. Zong L, Sun Q, Zhang H, Chen Z, Deng Y, Li D and Zhang L: Increased expression of circRNA_102231 in lung cancer and its clinical significance. Biomed Pharmacother 102: 639-644, 2018.

35. Zhang J, Zhao X, Zheng $X$ and Li F: Circular RNA hsa circ_0023404 exerts an oncogenic role in cervical cancer through regulating miR-136/TFCP2/YAP pathway. Biochem Biophys Res Commun 501: 428-433, 2018.

36. Hu C, Wang Y, Li A, Zhang J, Xue F and Zhu L: Overexpressed circ_0067934 acts as an oncogene to facilitate cervical cancer progression via the miR-545/EIF3C axis. J Cell Physiol 234: 9225-9232, 2019

37. Wang J, Li H and Liang Z: circ-MYBL2 serves as a sponge for miR-361-3p promoting cervical cancer cells proliferation and invasion. Onco Targets Ther 12: 9957-9964, 2019.

38. Chen R, Mao L, Shi R, Wang W and Cheng J: circRNA MYLK accelerates cervical cancer via Up-Regulation of RHEB and activation of mTOR signaling. Cancer Manag Res 12: 3611-3621, 2020.

39. Wang Y, Wang L, Wang W and Guo X: Overexpression of circular RNA hsa_circ_0001038 promotes cervical cancer cell progression by acting as a ceRNA for miR-337-3p to regulate cyclin-M3 and metastasis-associated in colon cancer 1 expression. Gene 733: 144273, 2020.

40. Panda AC, Abdelmohsen K, Martindale JL, Di Germanio C, Yang X, Grammatikakis I, Noh JH, Zhang Y, Lehrmann E, Dudekula DB, et al: Novel RNA-binding activity of MYF5 enhances Ccnd1/Cyclin D1 mRNA translation during myogenesis. Nucleic Acids Res 44: 2393-2408, 2016.

41. Mazloomian A, Araki S, Ohori M, El-Naggar AM, Yap D, Bashashati A, Nakao S, Sorensen PH, Nakanishi A, Shah S and Aparicio S: Pharmacological systems analysis defines EIF4A3 functions in cell-cycle and RNA stress granule formation. Commun Biol 2: 165, 2019.

42. Zheng X, Huang M, Xing L, Yang R, Wang X, Jiang R, Zhang L and Chen J: The circRNA circSEPT9 mediated by E2F1 and EIF4A3 facilitates the carcinogenesis and development of triple-negative breast cancer. Mol Cancer 19: 73, 2020.

43. Xu B, Yang N, Liu Y, Kong P, Han M and Li B: Circ_csell Inhibits Colorectal Cancer Proliferation by Binding to eIF4A3. Med Sci Monit 26: e923876, 2020.

44. Sun HD, Xu ZP, Sun ZQ, Zhu B, Wang Q, Zhou J, Jin H, Zhao A, Tang WW and Cao XF: Down-regulation of circPVRL3 promotes the proliferation and migration of gastric cancer cells. Sci Rep 8: 10111, 2018.

45. Tang W, Wang D, Shao L, Liu X, Zheng J, Xue Y, Ruan X, Yang C, Liu L, Ma J, et al: LINC00680 and TTN-AS1 stabilized by EIF4A3 promoted malignant biological behaviors of glioblastoma cells. Mol Ther Nucleic Acids 19: 905-921, 2020.

46. Shibuya T, Tange TØ, Sonenberg N and Moore MJ: eIF4AIII binds spliced mRNA in the exon junction complex and is essential for nonsense-mediated decay. Nat Struct Mol Biol 11: 346-351, 2004

This work is licensed under a Creative Commons Attribution-NonCommercial-NoDerivatives 4.0 International (CC BY-NC-ND 4.0) License. 